\title{
Monitoração intraoperatória com teste de estimulação eletromiográfica dos instrumentais de pacientes submetidos à correção cirúrgica de escoliose idiopática
}

Intraoperative neurophysiological monitoring of patients undergoing idiopathic scoliosis surgical correction

Seguimiento de estimulación intraoperatoria con electromiografía de los instrumentales de pacientes sometidos a corrección quirúrgica de la escoliosis idiopática

\author{
Wagner Pasqualini ${ }^{1}$ \\ Marcos Antonio Tebet ${ }^{2}$ \\ Marcio Oliveira Penna de Carvalho ${ }^{3}$ \\ Rubens Massaru Kanno ${ }^{4}$ \\ Alfredo Torres Castellon ${ }^{5}$
}

\section{RESUMO}

Objetivo: apresentar a metodologia empregada na monitoração neurofisiológica de pacientes submetidos a tratamento para correção de escoliose idiopática com instrumentação cirúrgica, utilizando parafusos pediculares torácicos e lombares. Métodos: foram estudados, retrospectivamente, 32 prontuários de pacientes operados no Serviço de Cirurgia da Coluna Vertebral do Instituto da Coluna,Jundiaí, São Paulo, entre os anos de 2004 e 2008. A idade variou de 11 a 18 anos, e a maioria era do sexo feminino (93,75\%). Resultados: verificou-se que em seis pacientes houve relação entre a incidência de positividade no teste com estimulação eletromiográfica dos instrumentais e mau posicionamento dos parafusos pediculares, sem evidências de complicações após

\section{ABSTRACT}

Objective: to present the methodology used for neurophysiological monitoring of patients undergoing idiopathic scoliosis correction with surgical instrumentation, using thoracic and lumbar pedicle screws. Methods: retrospective study of the medical records of 32 patients submitted to surgery at the Spine Surgery Service of Instituto da Coluna, Jundiaí, São Paulo, Brazil, from 2004 to 2008. Ages ranged from 11 to 18 years, and the majority of the patients was female (93.75\%). Results: a correlation between positive clinical findings and pedicle screw malposition was found in six patients submitted to electromyographic stimulation instruments test, with no evidence of complications after correction of

\section{RESUMEN}

Objetivo: presentar la metodología utilizada en la vigilancia neurofisiológica de los pacientes sometidos al tratamiento de corrección de la escoliosis idiopática, con el uso de instrumentación quirúrgica, utilizando tornillos pediculares torácicos y lumbares. Métodos: se estudiaron 32 pacientes operados en el Servicio de Cirugía del Raquis del Instituto de la Columna (Jundiai), en el Brasil, entre 2004 y 2008. Las edades oscilaban entre los 11 a 18 años y la mayoría era mujeres (93,75\%). Resultados: se ha detectado en seis pacientes la relación entre la incidencia de positivo en la prueba de estimulación con electromiografía de los instrumentales y la mala colocación de los tornillos pediculares, sin evidencias de complicaciones después de la correc-

\footnotetext{
Trabalho realizado no Serviço de Cirurgia da Coluna Vertebral do Instituto da Coluna - IC - Jundiaí (SP), Brasil.

'Mestre; Professor Colaborador da Universidade Estadual de Campinas - UNICAMP - Campinas (SP), Brasil.

2Doutor; Professor Adjunto da Faculdade de Medicina de Jundiaí - FMJ - Jundiaí (SP), Brasil.

${ }^{3}$ Mestre; Médico Ortopedista e Cirurgião da Coluna do Instituto da Coluna - IC - Jundiaí (SP), Brasil.

${ }^{4}$ Médico Ortopedista; Cirurgião da Coluna do Instituto da Coluna - IC - Jundiaí (SP), Brasil.

${ }^{5}$ Fisiologista; Mestre em Ciências Médicas pela Faculdade de Ciências Médicas da Santa Casa de São Paulo - FCMSCSP - São Paulo (SP), Brasil.
} 
correção dos mesmos. Conclusão: os resultados confirmam a eficácia da monitoração intraoperatória. screw position. Conclusion: results confirm the efficacy of intraoperative monitoring. ción de los mismos. Conclusión: los resultados confirman la eficacia de la monitorización intraoperatoria.
DESCRITORES: Monitorização intra-operatória; Escoliose; Eletromiografia; Parafusos ósseos; Neurofisiologia; Potencial evocado motor
KEYWORDS: Monitoring, intraoperative; Scoliosis; Electromyography; Bone screws; Neurophysiology; Evoked potentials, motor
DESCRIPTORES: Monitoreo intraoperatorio; Escoliosis; Electromiografía; Tornillos óseos; Neurofisiología; Potenciales evocados motores

\section{INTRODUÇÃO}

A cirurgia para correção da escoliose idiopática tem se tornado tratamento de escolha ${ }^{1}$. Nos últimos anos, a instrumentação cirúrgica com parafusos pediculares vem sendo utilizada para o tratamento da escoliose, o que tem aumentado o risco de dano neurológico que, apesar de raro $(0,06 \%)^{1}$, quando ocorre é catastrófico.

A monitorização eletrofisiológica é atualmente um cuidado primário que permite a detecção precoce e a reversão de muitas complicações neurológicas ${ }^{2-4}$.

A primeira descrição de uma técnica de potencial evocado motor (PEM) foi apresentada por Owen et al..$^{5}$, em 1988. O uso do potencial evocado somatossensitivo (PESS) é de particular interesse nos casos de escoliose neuromuscular ${ }^{6}$ e com a presença de seringomielia associada, mas tem sido comprovada sua valia nas cirurgias de escoliose idiopática ${ }^{3}$.

Não há consenso na literatura sobre a utilização conjunta do PESS, PEM e estimulação eletromiográfica (EMG) aplicada diretamente nos instrumentais.

O objetivo deste estudo foi apresentar a metodologia empregada na monitorização neurofisiológica por EMG de pacientes submetidos a tratamento para correção de escoliose idiopática com instrumentação cirúrgica, utilizando estimulação eletromiográfica dos instrumentais e parafusos pediculares torácicos e lombares.

\section{MÉTODOS}

Foram analisados 32 pacientes $(93,75 \%$ do sexo feminino e $6,25 \%$ do masculino) com média de idade de 14,7 anos. Os pacientes foram submetidos ao tratamento cirúrgico, por via posterior, para correção de escoliose idiopática sob monitoração de EMG intraoperatória, entre os anos de 2004 e 2008.

Todos os pacientes foram avaliados no pré-operatório e classificados como "E" na escala de Frankel.

Nenhum dos pacientes recebeu medicação pré-anestésica. A monitoração na sala de cirurgia constou de eletrocardioscopia, frequência cardíaca, saturação arterial periférica de oxigênio, pressão arterial não-invasiva e capnografia.

Foi utilizada anestesia endovenosa com o intuito de interferir o mínimo possível nas respostas ${ }^{7}$ da EMG. A ventilação foi mantida e controlada mecanicamente por volume ou pressão.
Os seguintes fármacos e respectivas dosagens foram utilizados durante o ato cirúrgico:

- propofol - indução: 1,0 a 2,5 miligramas/quilograma de peso/minuto; manutenção: 50 a $200 \mu \mathrm{g} / \mathrm{kg}$ de peso/ minuto;

- alfentanila - indução: 30 a $50 \mu \mathrm{g} / \mathrm{kg}$ de peso/minuto; manutenção: 0,5 a $1,5 \mu \mathrm{g} / \mathrm{kg}$ de peso/minuto.

Não foi utilizado relaxante muscular, exceto nos pacientes em que houve entubação endotraqueal difícil, quando então foram aplicadas drogas curarizantes de ação rápida (menos de 30 minutos de ação) em dose única, para facilitar o procedimento anestésico da entubação endotraqueal. No final da cirurgia, o paciente foi acompanhado até a sala de recuperação pós-anestésica. $\mathrm{O}$ exame clínico neurológico foi revisado após 24 horas e na ocasião da alta hospitalar.

Foi realizada EMG com estimulação direta dos parafusos pediculares nos pacientes submetidos à instrumentação com o uso desse tipo de parafusos como elementos de fixação. Para a realização desses procedimentos, foi usado um analisador neurofisiológico modelo Keypoint $\left(\right.$ Meditronic $^{\circledR}$ ), quatro canais, software 3.21 e um estimulador modelo D185 (Digitimer ${ }^{\circledR}$ ).

A monitoração do posicionamento de parafuso transpedicular foi realizada por EMG, com captação por eletrodos de superfície modelo 9013L0452 (Meditronic ${ }^{\circledR}$ ), posicionados nos músculos representativos dos miótomos a serem monitorados na colocação dos $\operatorname{parafusos}^{8}$ (Tabela 1). A estimulação elétrica catódica foi feita por estimulador de vareta referenciado a ânodo do tipo agulha, localizada em partes moles, sendo o estímulo de $10 \mu \mathrm{A}$ de intensidade e duração de 0,2 ms, com frequência de estímulo de $3 \mathrm{~Hz}$. Considerou-se alterada a resposta quando houve presença de resposta muscular após estimulação do parafuso ${ }^{8}$

$\mathrm{O}$ resultado foi baseado na presença ou ausência de alterações neurológicas, como déficit pós-operatório ou alteração na EMG, causada pela manobra cirúrgica, sendo esta corrigida no ato cirúrgico. Manobra cirúrgica foi considerada como sendo todo procedimento realizado pelo cirurgião durante a cirurgia, como passagem dos parafusos e correção da deformidade.

Entende-se como resultado da monitoração na EMG o resultado registrado pelo sistema de neuromonitoração. 
Um resultado negativo na monitoração na EMG foi determinado quando a manobra cirúrgica não ocasionou queda da amplitude abaixo do nível de advertência. Um resultado positivo na monitoração foi determinado quando a manobra cirúrgica ocasionou queda da amplitude abaixo do nível de advertência. O sucesso da neuromonitoração em detectar déficit neurológico ocasionado pela cirurgia pode ser expresso em valores de falso-positivo e falso-negativo.

\section{RESULTADOS}

Não houve a ocorrência de falso-negativo e de falso-positivo no grupo de 32 pacientes estudados; todos foram classificados como Frankel E no pós-operatório.

O teste de despertar não foi utilizado nas cirurgias realizadas.

Encontramos relação entre a incidência de positividade no teste com EMG em seis pacientes, o que foi atribuído ao mau posicionamento de parafusos pediculares, o qual foi prontamente corrigido, e o estudo neurofisiológico retornou ao normal.

\section{DISCUSSÃO}

A monitoração intraoperatória utilizando o PESS tem se tornado habitual em muitos centros médicos, diminuindo a incidência de lesão neurológica pós-operatória ${ }^{3}$. No entanto, a radiculopatia pós-operatória tem sido a complicação mais comum da cirurgia toracolombar, particularmente em pacientes submetidos à instrumentação segmentar, na qual a incidência pode chegar a mais de $10 \%{ }^{2}$.

Escolhemos como referência o PEM e o estímulo com EMG durante as etapas de instrumentação e correção das curvas escolióticas, porque são mais sensíveis ao comprometimento neurológico antes mesmo do $\mathrm{PESS}^{4,5}$. Considerando-se o tempo necessário para detectar as disfunções dos elementos neurais espinais, os potenciais evocados somatossensitivos mostram uma disfunção aproximadamente 11 minutos após ter acontecido a alteração da via somatossensitiva. Os PEMs musculares nos mostram alterações nos tratos corticoespinais entre 1 e 2 minutos. As alterações apresentadas na eletromiografia após a estimulação do parafuso pedicular apresentaram-se imediatamente após o estímulo (lembrando a pouca especificidade dessa resposta na tentativa de determinar se a lesão é de via sensitiva, motora ou de raiz neural). Essa técnica, no entanto, não garante informações precisas em todos os casos; as respostas podem ser facilmente influenciadas por vários agentes anestésicos, mesmo com o uso de avançados métodos de estimulação ${ }^{9,10}$. Salientamos a importância do protocolo de anestesia, os cuidados com hipotensão e o uso de eletrocautério, que podem influenciar a resposta da monitoração.

MacEwen et al. ${ }^{11}$ apresentaram na Scoliosis Research Society (SRS), em 1975, que a recuperação neurológica está diretamente correlacionada à rapidez com que o instrumental é retirado. Em nosso estudo, nos seis casos que apresentaram positividade, os parafusos foram retirados
TABELA 1 - Grupos musculares monitorados intraoperatoriamente durante o teste de EMG

\begin{tabular}{|c|c|}
\hline $\begin{array}{l}\text { Inervação da raiz } \\
\text { nervosa }\end{array}$ & Músculos monitorados e sua ação \\
\hline L2-L4 & Adutor magno \\
\hline L3;4 & Reto femoral* (quadríceps: extensão) \\
\hline \multirow[t]{2}{*}{$\llcorner 4 ; 5$} & Vasto lateral (abdução) \\
\hline & Tibial anterior* (dorsiflexão) \\
\hline \multirow[t]{2}{*}{ L5-S 1} & Bíceps femoral* (isquiotibiais) \\
\hline & Glúteo máximo (S1;L5) \\
\hline S1 & $\begin{array}{l}\text { Tríceps sural (gastrocnêmio e solear: } \\
\text { flexão plantar }\end{array}$ \\
\hline S2-S5 & Musculatura perineal* \\
\hline
\end{tabular}

*Músculos mais comumente utilizados.

e recolocados imediatamente, não havendo a ocorrência de déficit neurológico pós-operatório. Estamos de acordo com Pereon et al. ${ }^{12}$, que têm recomendado o uso de PEM como substituto do potencial sensitivo nas etapas mais importantes do procedimento cirúrgico, que são a locação de parafusos pediculares e a correção da escoliose.

O PESS do dermátomo é mais sensível que o PESS dos nervos mistos para a detecção da radiculopatia ${ }^{13}$ e tem sido usado para a monitoração das raízes lombossacrais. No entanto, o PESS do dermátomo fornece informações apenas da função da raiz do nervo sensitivo dorsal. Além disso, as respostas do PESS do dermátomo constituem um método demorado, possuem uma menor amplitude e necessitam de um sinal médio prolongado para reproduzir informações, principalmente em um ambiente eletricamente hostil, como uma sala cirúrgica ${ }^{2}$.

A monitorização eletromiográfica contínua (EMG) é mais sensível que o PESS do dermátomo para a detecção de radiculopatia ${ }^{13}$ e pode fornecer uma informação contínua, em tempo real, da função da raiz durante a cirurgia. Um aumento na frequência do potencial da unidade motora é sensível e específico para uma irritação mecânica brusca e/ou lesão da raiz do nervo. Consequentemente, a monitoração EMG pode fornecer um retorno contínuo para que o cirurgião possa determinar o local e a possibilidade de uma lesão da raiz nervosa² .

Apesar de a EMG estimulada poder ser utilizada na identificação de irritação ou lesão nervosa, ainda podem ocorrer danos, devido à utilização da EMG de forma intermitente. A técnica de estimulação pedicular através de instrumental constantemente eletrificado permite uma monitoração e, concomitantemente, a identificação dos pedículos comprometidos antes que ocorra uma lesão da raiz neural ${ }^{2,14}$. Os parafusos e furos posicionados corretamente apresentam uma parede pedicular com osso cortical separando-os da raiz nervosa; dessa forma, o osso cortical representa uma barreira de alta impedância à passagem de corrente elétrica ${ }^{2}$. Uma fratura da parede pedicular passa a expor a raiz nervosa (Figura 1). Um estímulo elétrico nesse furo ou parafuso ativa a raiz nervosa adjacente, causando 


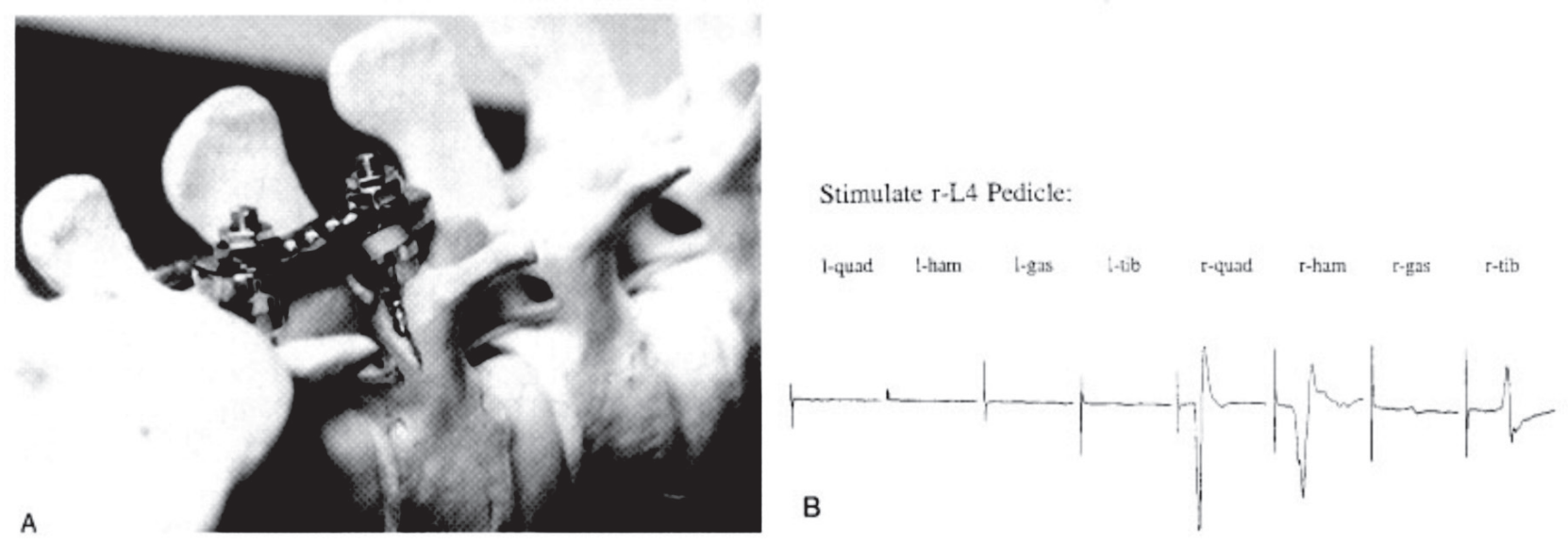

Figura 1

Quebra do pedículo. A: como a quebra do pedículo aparece com o instrumental no lugar. B: resposta eletromiográfica com corrente dispersa no pedículo quebrado. Os músculos ipsilaterais são estimulados devido à dispersão da corrente através dos tecidos moles.

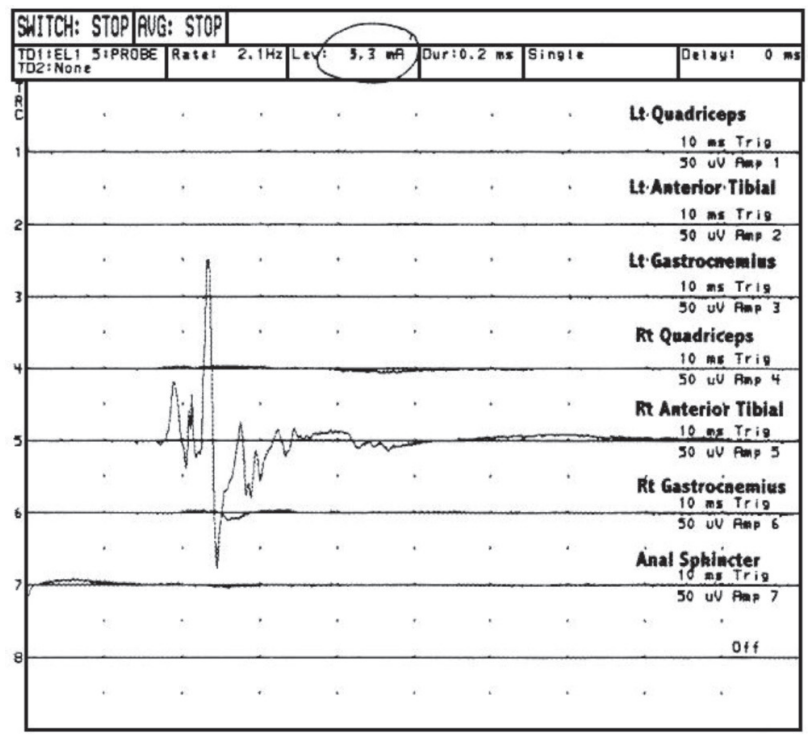

Figura 2

Registro eletromiográfico com estimulação do parafuso do lado direito em L5. A presença de uma resposta na musculatura tibial anterior direita à estimulação de baixa intensidade $(5,3 \mathrm{~mA})$ é sugestiva de quebra da cortical pelo parafuso transpedicular.

uma resposta no músculo do miótomo correspondente com um estímulo de menor intensidade, comparado com a situação em que a parede pedicular permanece intacta. Um limiar de estímulo menor que 4-5 mA é sugestivo de perfuração do osso cortical pela instrumentação transpedicular ${ }^{2}$ (Figura 2).

Verificamos que não houve falso-positivo nem falsonegativo na utilização da EMG estimulada. A atividade eletromiográfica pode ocorrer com a violação da cortical; isso não quer dizer que houve lesão da raiz nervosa, apesar de o risco existir ${ }^{14}$. Nesses casos, o parafuso pode ser redirecionado quando necessário, sem causar qualquer dano ou dor no pós-operatório. O falso-negativo pode ocorrer quando não existe resposta. Existem duas formas óbvias de isso ocorrer: uma relacionada ao aparelho e outra fisiológica ${ }^{14}$. A falha do equipamento no momento da cirurgia pode ser evitada com a verificação de eletrodos, conexões, estimuladores, computadores (hardware e software). O falso-negativo fisiológico pode incluir situações em que houve quebra do pedículo sem alterações na EMG. Essa falha não foi verificada neste nem em outros estudos.

Este estudo demonstrou a validade de nosso protocolo de monitoração. A combinação do PESS e PEM, associado à EMG estimulada, mostrou-se $100 \%$ sensível à presença de lesão neurológica.

Concordamos com as observações de Holland ${ }^{2}$, que afirma que a EMG pode fornecer informações em tempo real sobre a localização da raiz nervosa e sobre uma potencial lesão cirúrgica intraoperatória. A EMG confirma que a instrumentação intrapedicular está corretamente posicionada nos limites internos das corticais dos pedículos ${ }^{2}$.

A monitorização intraoperatória torna o teste do despertar desnecessário.

O sucesso da monitoração depende da boa comunicação e cooperação entre o cirurgião, o anestesista e o neurofisiologista.

Este é um estudo preliminar que nos estimulou a iniciar uma linha de pesquisa do uso do PESS, do PEM e da EMG nas cirurgias de correção de deformidades da coluna vertebral.

\section{CONCLUSÃO}

A monitorização intraoperatória com EMG estimulada mostrou-se útil e realizável quando aplicada a pacientes submetidos à correção cirúrgica de escoliose idiopática por instrumentação com parafusos pediculares torácicos e lombares, o que permite verificar se o parafuso encontra-se muito próximo da raiz nervosa, possibilitando, caso necessário, a correção da distância entre parafuso e raiz. 


\section{REFERÊNCIAS}

1. Hicks JM, Singla A, Shen FH, Arlet V. Complications of pedicle screw fixation in scoliosis surgery: a systematic review. Spine (Phila Pa 1976). 2010:35(11):E465-70.

2. Holland NR. Intraoperative electromyography during thoracolumbar spinal surgery. Spine (Phila Pa 1976). 1998;23(17):1915-22.

3. Nuwer MR, Dawson EG, Carlson LG, Kanim LEA, Sherman JE. Somatosensory evoked potential spinal cord monitoring reduces neurological deficits after scoliosis surgery: results of a large multicenter survey. Electroencephalography and Clinical Neurophysiology. 1995;96(1):6-11.

4. Accadbled F, Henry P, de Gauzy JS, Cahuzac JP. Results of a prospective, consecutive series of 191 cases. Spine (Phila Pa 1976). 2006;31(22):2614-23.

5. Owen JH, Laschinger J, Bridwell K, Shimon S, Nielsen C, Dunlap $\mathrm{J}$ et al. Sensitivity and specificity of somatosensory and neurogenicmotor evoked potentials in animals and humans. Spine (Phila Pa 1976). 1988;13(10):1111-8.

6. Noordeen MH, Lee J, Gibbons CE, Taylor BA, Bentley G. Spinal cord monitoring in operations for neuromuscular scoliosis. J Bone Joint Surg Br. 1997;79(1):53-7.
7. Taniguchi M, Nadstawek J, Pechstein U, Schramm J. Total intravenous anesthesia for improvement of intraoperative monitoring of somatosensory evoked potentials during aneurysm surgery.

Neurosurgery. 1992;31(5):891-7.

8. Calancie B, Madsen P, Lebwohl N. Stimulus-evoked EMG monitoring during transpedicular lumbosacral spine instrumentation. Initial clinical results. Spine (Phila Pa 1976). 1994;19(24):2780-6.

9. Frei FJ, Ryhult SE, Duitmann E, Hasler CC, Luetschg J, Erb TO. Intraoperative monitoring of motor-evoked potentials in children undergoing spinal surgery. Spine (Phila Pa 1976). 2007;32(8):911-7

10.Kawaguchi M, Sakamoto T, Inoue S, Kakimoto M, Furuya H, Morimoto $\mathrm{T}$ et al. Low dose propofol as a supplement to ketamine-based anesthesia during intraoperative monitoring of motor-evoked potentials. Spine (Phila Pa 1976). 2000;25(8):974-9.

11. MacEwen GD, Bunnell WP, Sriram K. Acute neurological complications in the treatment of scoliosis. A report of the Scoliosis Research Society. J Bone Joint Surg Am. 1975;57(3):404-8.
12.Péréon Y, Nguyen The Tich S, Delécrin J, Passuti N. Somatosensoryand motor-evoked potential monitoring without a wake-up test during idiopathic scoliosis surgery: an accepted standard of care. Spine (Phila Pa 1976). 1999;24(11):1169-70.

13.Aminoff MJ, Goodin DS, Parry GJ, Barbaro NM, Weinstein PR, Rosenblum ML. Electrophysiologic evaluation of lumbosacral radiculopathies: electromyography, late responses, and somatosensory evoked potentials. Neurology. 1985;35(10):1514-8.

14.Rose RD, Welch WC, Balzer JR, Jacobs GB. Persistently electrified pedicle stimulation instruments in spinal instrumentation. Technique and protocol development. Spine (Phila $\mathrm{Pa}$ 1976). 1997;22(3):334-43.

\section{Correspondência}

Wagner Pasqualini

Rua Abílio Figueiredo, 92, sala 91 -

Anhangabaú

CEP: 13208-140 - Jundiaí (SP), Brasil.

E-mail:drwagner@institutodacoluna.com.br 